\title{
Tendencias y proyecciones de la fuerza de trabajo del Área Metropolitana del Valle de Aburrá, 2010-2030 ${ }^{1}$
}

Jorge E. Horbath. El Colegio de la Frontera Sur (Ecosur), Unidad Chetumal, México

RESUMEN | Las proyecciones de la fuerza de trabajo constituyen el ejercicio demográfico más cautivador y más complejo de realizar, dados los fenómenos imbricados en dicho proceso. Destacan al respecto aquellos vinculados a los cambios generacionales, específicamente el paso desde población en edad infantil a población en edad de trabajar, y posteriormente a población económicamente activa. Al realizar tales proyecciones para el Área Metropolitana del Valle de Aburrá (la principal área metropolitana de Colombia), se encuentra una gran dinámica económica entretejida con otros fenómenos sociales, como la violencia y la presencia de jóvenes que ni estudian ni trabajan (conocidos como "Ninis"), junto a cambios territoriales de distintas tendencias en las zonas tanto urbanas como rurales de Medellín, y en los municipios que integran el área. En particular, este artículo muestra las proyecciones de población y fuerza de trabajo en el marco del proyecto Plan Director BIO-2030.

PAlabras ClaVe | demografía, mercado de trabajo, áreas metropolitanas.

abstract | Developing demographic projections of the labor force is one of the most captivating and complex tasks in contemporary urban study, due to the demographic phenomena interwoven in such a process. Paramount among such phenomena are those aspects related to generational changes, as people grow up from constituting an infant population, to reaching working age and, later, to be economically active. In making such projections for the Aburrá Valley (the main me-tropolitan area of Colombia), strong economic dynamics can be identified that are interwoven with other phenomena, such as violence, and youth that neither study nor work (known as "neet": Not in Employment, Education or Training), and different trends of territorial changes both in urban and rural areas of Medellin and the surrounding municipalities. Specifically, this article presents population and workforce projections in the framework of the BIO-2030 Master Plan project.

KEY WORDS | demography, labour market, metropolitan areas.

Recibido el 26 de febrero de 2013, aprobado el 1 de octubre de 2013

E-mail: jhorbath@gmail.com

Correspondencia: El Colegio de la Frontera Sur Unidad Chetumal Av. Centenario km 5.5, CP 77014, Chetumal, Quintana Roo. AP 424, México

1 Documento síntesis del informe titulado "Dinámicas poblacionales en el Área Metropolitana de Medellín-Valle de Aburrá”, elaborado por el autor para el proyecto Plan Director BIO-2030, coordinado por el Centro de Estudios Urbanos y Ambientales (URBAM) de la Universidad EAFIT, la entidad administrativa Área Metropolitana del Valle de Aburrá y la Alcaldía de Medellín. 


\section{Introducción}

El estudio de las dinámicas poblacionales de un territorio a través de la aplicación formal de las técnicas utilizadas en la construcción de un modelo demográfico, implica adentrarse en el contexto de dichas dinámicas y analizar sus tendencias, así como sus efectos en la ocupación espacial del territorio, sus modificaciones y el encadenamiento de los fenómenos que las constituyen. Ello sustentará las explicaciones relativas a la transformación del espacio y permitirá vislumbrar los retos futuros en cuanto a planeación y planificación del desarrollo, para hacer frente a los problemas de cambios poblacionales y el comportamiento de las variables demográficas, anticipando sus efectos.

Después de las proyecciones de la población total, las más interesantes son las proyecciones de la fuerza de trabajo, debido a que responden a dinámicas generacionales cruzadas. No solamente contienen las propias tendencias de las distintas generaciones, sino también los cambios que registran las trayectorias de vida de sus integrantes, rebasando así los umbrales demográficos para observarlas en su condición laboral. Realizar las proyecciones de la fuerza de trabajo entre los años 2010 y 2030 para la segunda y más dinámica área metropolitana de Colombia -el Área Metropolitana del Valle de Aburrá- condensa el apremiante reto de incorporar en ellas los efectos demográficos generados por dos variables fundamentales para dicha región: la mortalidad y la migración. En la primera, conocida como la máxima condensadora del fenómeno de la violencia, se integran las estadísticas de muertes violentas; la segunda, por su parte, se encuentra vinculada al mismo fenómeno social, el de la violencia, esta vez al efecto que ella tiene en el territorio como causa del desplazamiento de la población. Tal esfuerzo de análisis poblacional ha sido posible al realizarse el proyecto Plan Director BIO-2030, coordinado por el Centro de Estudios Urbanos y Ambientales urbam de la Universidad EAfit, la entidad administrativa Área Metropolitana del Valle de Aburrá y la Alcaldía de Medellín, en el que se hicieron las proyecciones de población y fuerza de trabajo para el área metropolitana. El Área Metropolitana del Valle de Aburrá está conformada por diez municipios: Medellín, fundada en 1616; Copacabana, en 1615; Girardota, en 1620; Bello, en 1676; La Estrella, en 1685; Itagüí, en 1743; Envigado, en 1775; Barbosa, en 1795; Caldas en 1840 y Sabaneta en 1903 (Departamento Administrativo de Planeación de Medellín [DAP], 2002).

El documento se inicia con los principales resultados de las proyecciones totales de población, base de las tendencias demográficas en el área metropolitana; luego se muestran los resultados de las proyecciones de la fuerza de trabajo, tanto de la Población en Edad de Trabajar (PET) como de la Población Económicamente Activa (PEA) y sus componentes. Posteriormente se expone uno de los nuevos fenómenos demográficos contemporáneos en América Latina, como es la magnitud y tendencia del grupo de los jóvenes que ni estudian ni trabajan (más conocidos en la literatura especializada como "Ninis"), respecto del cual no es ajena el Área Metropolitana del Valle de Aburrá. Después se expone el comportamiento espacial de la fuerza de trabajo y sus resultados en el marco de análisis de los contornos de crecimiento en el área metropolitana; se finaliza con las estimaciones de la ocupación y el desempleo, 
contemplando los escenarios posibles del crecimiento de la fuerza de trabajo de acuerdo con los comportamientos históricos de la economía regional y las expectativas económicas futuras.

\section{Tendencias y proyecciones de población 2010-2030}

Los efectos de la "transición demográfica", identificada hace más de setenta años en gran parte de los países desarrollados y en desarrollo, marca un proceso de notable disminución de los altos niveles de mortalidad y fecundidad a nivel mundial existentes hasta entonces. El efecto del momento de reemplazo generacional en el que cada mujer tendría dos hijos (Notestein, 1945; Leibenstein, 1957), resulta en un cambio en la estructura por edades (Miró, 2003) y, consecuentemente, en la diferencia de la distribución etaria en los decesos, que ahora se concentran en la población de mayor edad (Janssen \& Kunst, 2004). Estos resultados mostraron similitudes tanto en el inicio como en la culminación de dicha transición (Heligman, Chen \& Babakol, 1993), aun cuando los tiempos en que se dieron los procesos, así como su intensidad y rapidez, presentaron importantes diferencias regionales, e incluso hacia el interior de los países involucrados. Esta dinámica en la natalidad y la mortalidad tiene su correlato en la modernización económica, la industrialización, flujos de urbanización y transformaciones sociales. La posibilidad de contar con bienes y servicios básicos trajo la reducción de muertes causadas por infecciones, produciendo un cambio en la composición de la mortalidad al incrementarse las causas de mortalidad por padecimientos genéticos (Omran, 1971 y 1998). Hay, entonces, una relación entre la transición demográfica y la transición epidemiológica (Frenk, Bobadilla, Stern, Frejka \& Lozano, 1991a; Galyin \& Kates, 1997), pues las tasas de sobrevivencia mayores determinan un crecimiento en la proporción de mujeres, jóvenes y niños en la población total, que queda expuesta a las enfermedades crónicas y el proceso degenerativo natural. Esta mirada ha recibido fuertes críticas que consideran como una debilidad teórica el hecho de que el inicio y culminación de los procesos de equilibrio y reemplazo entre mortalidad y morbilidad no sean claros (Mackenbach, 1994; Bolaños, 2000).

De los factores que aportan a la transformación de la sociedad, el cambio epidemiológico es un factor concluyente. Más allá de dar un término formal a las modificaciones en la distribución de las enfermedades y la mortalidad en relación a la causalidad y sus efectos en el tiempo, los avances en el tratamiento de enfermedades cardiovasculares fueron considerados como una cuarta fase de la transición epidemiológica (Olshanky \& Ault, 1986; Roger \& Hackemberg, 1987), pero en la actualidad se los ve como resultantes de la acción de la población misma en pos de mejorar sus condiciones sanitarias (Caldwell, 1990; Frenk et al., 1991a; Meslé \& Vallin, 2006) y serían una transición en la salud. Sin embargo, la transición epidemiológica no designa solamente un avance hacia un estadio positivo, sino también hacia un rezago en el desarrollo ocasionado por procesos anormales y desequilibrados de industrialización, consumo extensivo e intensivo y urbanización acelerada, que generan altos niveles de contaminación, accidentes -tanto en el lugar de trabajo como fuera de él-, malos hábitos alimentarios, adicciones al 
tabaco, el alcohol y las drogas, además del aumento de trastornos mentales (Frenk et al., 1991b). Es evidente que las regiones más desarrolladas -que ya atravesaron las etapas de transición descritas- enfrentan los problemas de un avance desmesurado y que su experiencia puede servir a las regiones en desarrollo, como el Área Metropolitana del Valle de Aburrá y sus municipios, que recién están transitando ese proceso.

\section{Metodología y fuentes de información}

Las proyecciones para el Área Metropolitana del Valle de Aburrá contemplan las proyecciones de población desde 2006 hasta 2020 realizadas por el Departamento Administrativo Nacional de Estadística (DANE) para los diez municipios de esa área, ya que corresponden a datos oficiales provenientes del órgano institucional que administra la información estadística, y principalmente el acceso a los censos de población. Para las comunas y corregimientos de Medellín se cuenta con proyecciones desde 2006 hasta 2015 (y estimaciones para 1993 y 2005).

Para efectuar dichas proyecciones se utilizó el método de Relación de Cohortes, con el aplicativo prodem, y la aplicación del Instituto de Geografía y Estadística de Brasil (IBGE) conocida como Peq-AR, versión 2.0, que incluye el módulo Rcoortes para proyecciones anualizadas de población para áreas menores por sexo y edades, con la metodología por relación de cohortes para un número significativamente mayor de municipios. En los supuestos con que operan estas proyecciones oficiales, los factores diferenciales de crecimiento $\mathrm{K}$ son constantes dentro de cada grupo etario y se reducen a escala anual, pero no se consideran constantes las distribuciones relativas de las tasas específicas de fecundidad, por lo que varían para cada año que abarca el periodo que se proyectará (se adopta la evolución anual del patrón de fecundidad del departamento). Los parámetros de fecundidad (tasa global y específica) y de mortalidad (relación de sobrevivencia al nacer) fueron utilizados como punto medio de cada periodo de proyección municipal, que demandó una proyección departamental basada en el método de componentes (usado en el módulo Rcoortes), permitiendo a su vez la intervención de una metodología exógena para el cálculo de los datos para la cabecera de los municipios (DANE, 2008).

Tomando en cuenta estos elementos y considerando las tendencias marcadas por las proyecciones oficiales, para las nuevas proyecciones de 2020 a 2030 se aplicó la metodología de crecimiento escalonado de los grupos quinquenales de población por sexo, municipio y zona, desagregando en el caso de Medellín. Las estimaciones no fueron homogéneas para todas las unidades territoriales debido a que las proyecciones para municipios llegaban hasta 2020 y las proyecciones para comunas y corregimientos de Medellín, hasta 2015. Ello implicó tomar de plataforma los cinco años de 2016 a 2020 de las proyecciones de Medellín y descomponerla en las comunas y municipios. Una vez empatadas las series de población, se estimó la década de 2021 a 2030. Con esta metodología se introducen las dinámicas de los componentes demográficos contemplados por DANE (2007) en sus proyecciones municipales a 2020, aplicando funciones logísticas quinquenales en el tiempo y contrastándolas en las cohortes de los periodos que dan lugar a la diagonal en los diagramas de Lexis (Naciones Unidas, 2005) y funciones similares para el primero y el último grupo poblacional (menos de 5 y mayores de 80 ańos, respectivamente). 
Una vez obtenidas las proyecciones de la población en las estimaciones que empatan las proyecciones del DANE hasta 2020 con las estimaciones para el periodo 2020-2030, se procede a realizar las proyecciones de la Fuerza de Trabajo. Se reconoce, entonces, que la tendencia del número de activos depende tanto del volumen y estructura de la población en edad activa, como de la propensión hacia la actividad de la población (Blanes, Gil \& Pérez, 1996, pp. 205-206). El primer componente se trae desde las proyecciones de población, en tanto que el restante se elabora desde la configuración de supuestos sobre las tendencias futuras de las tasas de actividad por sexo y edad, para cada fragmento territorial metropolitano (comunas urbanas y rurales, cabeceras municipales y resto).

\section{Las tendencias resultantes}

Viendo los resultados, se obtiene una estimación de 4.389 .585 personas en el área metropolitana, 844.883 personas más entre 2010 y 2030, a un ritmo de $1 \%$ anual, con crecimiento mayor en la zona urbana de los extremos de la región central (Bello, Envigado, Itagüí) que en Medellín (véase Gráfico 1). De acuerdo con sus dimensiones, esos tres municipios aportarían más de 344.000 personas adicionales durante el periodo; Bello sobrepasaría el medio millón de habitantes en 2030, en tanto que entre Itagüí y Envigado se registraría una población de 600.000 para ese mismo año. La población del área metropolitana muestra que el $93 \%$ de ella se concentra en solo el $28 \%$ del territorio, buena parte en la zona central conurbada (Moreno, 2005).

\section{GRÁfICo I | Proyecciones de población total, Área Metropolitana de Medellín- Valle de Aburrá, 1985-2030}

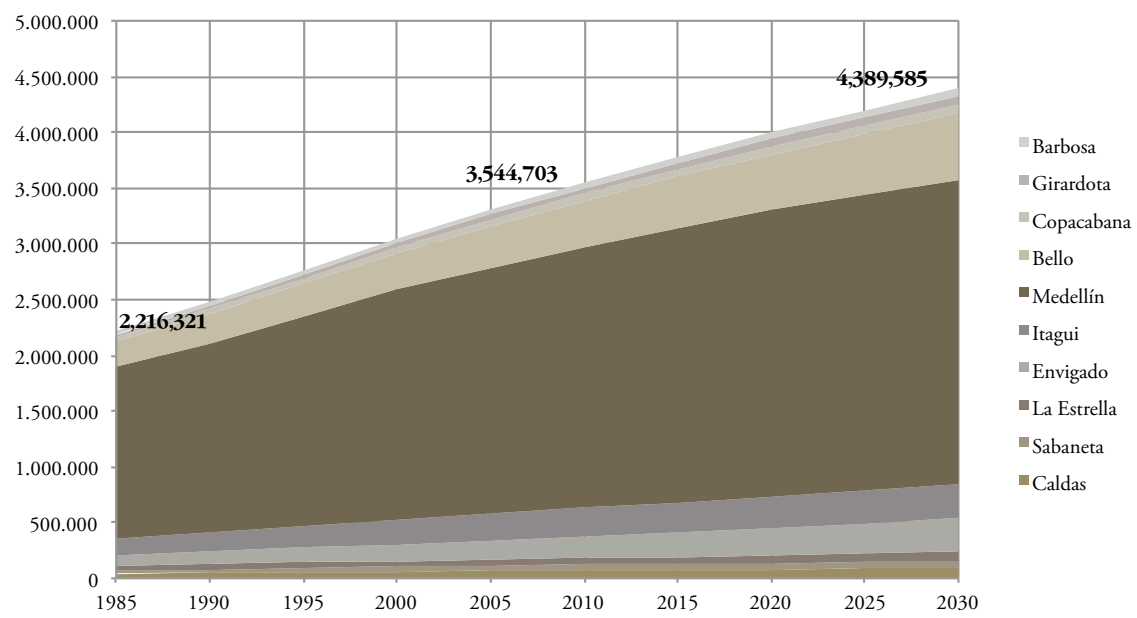

FUENTE CENSOS DE POBLACIÓN, Y PROYECCIONES MUNICIPALES DEL DANE Y MICRODATOS CENSALES DE IPUMS [INTEGRATED PUBLIC USE MICRODATA SERIES]-INTERNATIONAL, UNIVERSITY OF MINNESOTA. CÁLCULOS PROPIOS 
En términos de ritmo de crecimiento, se proyecta un aumento de más de 108.000 personas en el municipio de Envigado (un 54\% respecto a la población estimada para 2010), con lo que este sería el de mayor crecimiento, con un ritmo de 2,1\% (véase Cuadro 1). En los municipios adyacentes a la parte central del área, como Copacabana y La Estrella, que no cuentan con una población numerosa, se advierte un ritmo de crecimiento superior al promedio del área, aunque para el año 2030 sus habitantes no sobrepasarían los 100.000 habitantes. En los extremos de la región, Girardota tendría un crecimiento similar al de Envigado, ya que su población aumentaría 52,3\% respecto a la registrada en 2010, con un crecimiento anual del 2\%; y los municipios de Sabaneta y Barbosa, el 1,5 y 1,4\% respectivamente, mientras que el municipio de Caldas alcanzaría un ritmo de crecimiento similar al del promedio del área.

Son cuatro las etapas de metropolización en el poblamiento que plantea la teoría: i) urbanización, cuando la tasa de crecimiento porcentual anual de pobladores de la ciudad central supera a la de la periferia; ii) suburbanización, cuando la periferia alcanza una mayor tasa de crecimiento porcentual anual; iii) desurbanización, cuando en la cuidad se observa un despoblamiento relativo o absoluto; iv) reurbanización, cuando en la ciudad central ocurre un repoblamiento relativo o absoluto (Unikel, 1978; Garza, 2003; Sobrino, 2003; Busquets, 1993 y otros). El área metropolitana puede pasar de ser una región monocéntrica a policéntrica por la influencia de las fuerzas centrípeta de concentración y centrífuga de expansión, definiendo de esta manera las etapas mencionadas.

CUADro I | Retos de dinámica poblacional Área Metropolitana de MedellínValle de Aburrá, 2010-2030

\begin{tabular}{|c|c|c|c|c|c|c|c|c|c|}
\hline \multirow{2}{*}{ MUNICIPIO } & \multicolumn{2}{|c|}{ POBLACIÓN TOTAL } & \multicolumn{5}{|c|}{$2010-2030$} & \multicolumn{2}{|c|}{$\begin{array}{c}\% \text { POB. } \\
\text { CABECERA }\end{array}$} \\
\hline & 2010 & 2030 & Total & Var \% & $\begin{array}{l}\text { C. } \% \\
\text { Anual }\end{array}$ & Rural & Var \% & 2010 & 2030 \\
\hline Barbosa & 46.173 & 61.720 & 15.547 & 33,67 & 1,38 & 7.251 & 28,62 & 45,12 & 47,20 \\
\hline Girardota & 48.206 & 73.435 & 25.229 & 52,34 & 2,00 & 9.354 & 47,82 & 59,42 & 60,62 \\
\hline Copacabana & 65.773 & 87.851 & 22.078 & 33,57 & 1,38 & 1.628 & 18,73 & 86,78 & 88,25 \\
\hline Bello & 413.107 & 591.756 & 178.649 & 43,25 & 1,71 & -6.061 & $-61,39$ & 97,61 & 99,36 \\
\hline Medellín & 2.343 .049 & 2.724 .051 & 381.002 & 16,26 & 0,72 & -8.453 & $-25,15$ & 98,57 & 99,08 \\
\hline Itagui & 252.158 & 310.928 & 58.770 & 23,31 & 1,00 & 1.938 & 8,68 & 91,14 & 92,20 \\
\hline Envigado & 197.440 & 305.713 & 108.273 & 54,84 & 2,08 & -317 & $-3,88$ & 95,87 & 97,43 \\
\hline La Estrella & 57.437 & 77.878 & 20.441 & 35,59 & 1,45 & -3.059 & $-11,71$ & 54,51 & 70,38 \\
\hline Sabaneta & 48.264 & 66.302 & 18.038 & 37,37 & 1,51 & 2.103 & 21,30 & 79,54 & 81,93 \\
\hline Caldas & 73.096 & 89.952 & 16.856 & 23,06 & 0,99 & -99 & $-0,62$ & 78,02 & 82,25 \\
\hline Área Total & 3.544 .703 & 4.389 .586 & 844.883 & 23,84 & 1,02 & 4.287 & 2,39 & 94,93 & 95,81 \\
\hline
\end{tabular}

FUENTE CENSOS DE POBLACIÓN, Y PROYECCIONES MUNICIPALES DEL DANE Y MICRODATOS CENSALES DE IPUMS-INTERNATIONAL, UNIVERSITY OF MINNESOTA. CÁLCULOS PROPIOS 
Las proyecciones respecto a las zonas del área y sus municipios señalan que el fenómeno de ampliación de la mancha urbana se extiende: la población concentrada en la cabecera municipal pasaría de $94,9 \%$ a $95,8 \%$ en promedio, aumentando casi un punto porcentual en dos décadas. Este proceso -impulsado por Medellín- produce una gran transformación en la región central (Gaviria, 2009), donde los municipios aledaños a la capital del departamento registran una alta contracción de la población rural (tales los casos de Bello, Envigado y La Estrella [véase Cuadro 1]). A pesar de la expansión urbana que la región central impone a las zonas rurales de sus municipios, Itagüí todavía registraría incrementos de su población rural.

Vemos, entonces, que la configuración población-territorio se da en forma de anillos concéntricos, con Medellín en el núcleo de la región central que comparte con Bello y Envigado, mientras los demás municipios constituyen el anillo exterior. Las dinámicas entre población y ocupación del territorio están marcadas por el fenómeno de rururbanización, según lo muestran estudios como el de Muñoz (2010, pp. 358-359), Méndez, López y Márquez (2006), Villegas (2005, pp. 155-157) y Arroyave y Muńoz (1994), en que la actividad rural adquiere contenidos urbanos, dando lugar a nuevas configuraciones territoriales y sociales en el espacio metropolitano del Valle de Aburrá.

Gráfico 2 Proyecciones de la población total, comunas y corregimientos de Medellín, 2005-2030

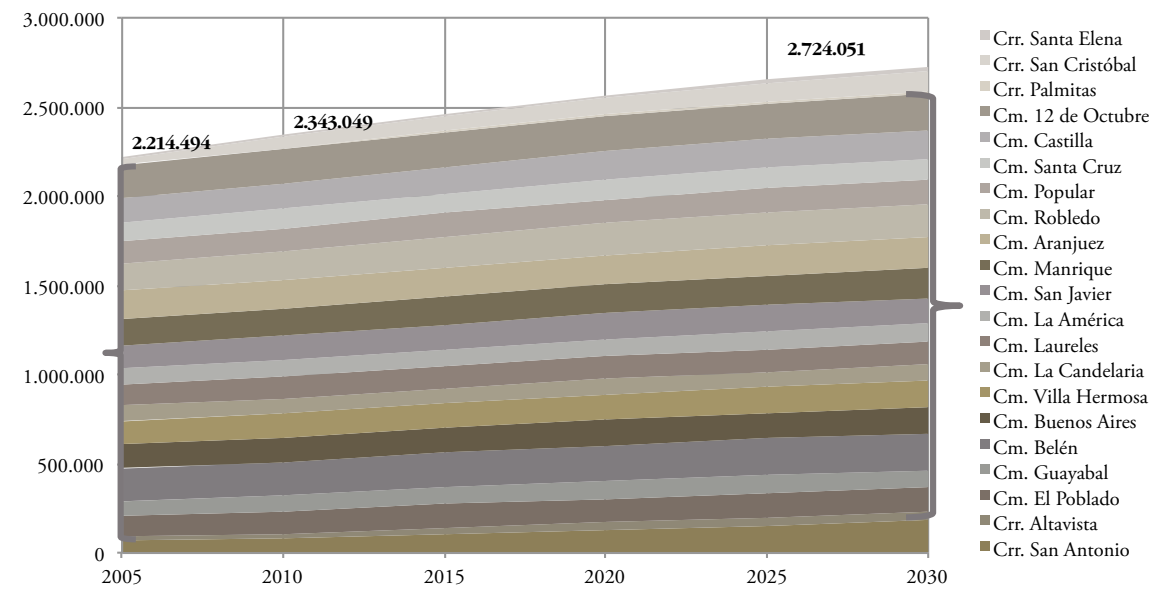

FUENTE CENSOS DE POBLACIÓN, Y PROYECCIONES MUNICIPALES DEL DANE Y MICRODATOS CENSALES DE IPUMS-INTERNATIONAL, UNIVERSITY OF MINNESOTA. CÁLCULOS PROPIOS

Vamos ahora a las proyecciones de población de Medellín que ostentan una dinámica especial: la estimación para 2030 es de 2.720 .051 habitantes con un aumento de 381.002 entre 2010 y 2030 (tasa de crecimiento anual de $0,72 \%$ ) y mayor crecimiento en los corregimientos que en las comunas (véase Gráfico 2), pero si bien hasta 2010 el municipio de Medellín concentraba el 65,7\% de la población del área (lo que se explica por el tamaño de su población y su dinámica socioproductiva), 
se espera que la fuerte dinámica de urbanización de los municipios adyacentes produzca una reducción a 62,8\% para 2030 (casi 3 puntos porcentuales). Asimismo, se avizoran grandes transformaciones en su composición territorial debido a que el crecimiento demográfico determinará un aumento de la población en las zonas rurales, producido por la búsqueda de nuevos territorios para establecerse (Zuluaga, 2000).

CUADRO 2 Dinámica poblacional en las comunas y corregimientos de Medellín, 2010-2030

\begin{tabular}{|c|c|c|c|c|c|}
\hline \multirow[t]{2}{*}{ MUNICIPIO } & \multicolumn{2}{|c|}{ POBLACIÓN TOTAL } & \multicolumn{3}{|c|}{$2010-2030$} \\
\hline & 2010 & 2030 & TOTAL & VAR $\%$ & c. $\%$ ANUAL \\
\hline Crr. Santa Elena & 14.501 & 22.829 & 8.328 & 57,43 & 2,16 \\
\hline Crr. San Cristóbal & 60.025 & 116.584 & 56.559 & 94,22 & 3,16 \\
\hline Crr. Palmitas & 4.370 & 11.402 & 7.032 & 160,91 & 4,57 \\
\hline Cm. 12 de Octubre & 190.155 & 204.732 & 14.577 & 7,67 & 0,35 \\
\hline Cm. Castilla & 146.471 & 158.474 & 12.003 & 8,19 & 0,38 \\
\hline Cm. Santa Cruz & 107.869 & 118.024 & 10.155 & 9,41 & 0,43 \\
\hline Cm. Popular & 126.887 & 137.829 & 10.942 & 8,62 & 0,39 \\
\hline Cm. Robledo & 163.559 & 182.613 & 19.054 & 11,65 & 0,52 \\
\hline $\mathrm{Cm}$. Aranjuez & 160.068 & 171.397 & 11.329 & 7,08 & 0,33 \\
\hline Cm. Manrique & 155.049 & 169.001 & 13.952 & 9,00 & 0,41 \\
\hline Cm. San Javier & 133.918 & 145.999 & 12.081 & 9,02 & 0,41 \\
\hline Cm. La América & 94.165 & 101.974 & 7.809 & 8,29 & 0,38 \\
\hline Cm. Laureles - Estadio & 120.607 & 129.200 & 8.593 & 7,13 & 0,33 \\
\hline Cm. La Candelaria & 85.000 & 90.311 & 5.311 & 6,25 & 0,29 \\
\hline Cm. Villa Hermosa & 134.235 & 145.375 & 11.140 & 8,30 & 0,38 \\
\hline Cm. Buenos Aires & 135.005 & 144.523 & 9.518 & 7,05 & 0,32 \\
\hline Cm. Belén & 193.343 & 207.947 & 14.604 & 7,55 & 0,35 \\
\hline Cm. Guayabal & 91.147 & 100.180 & 9.033 & 9,91 & 0,45 \\
\hline Cm. El Poblado & 120.695 & 138.242 & 17.547 & 14,54 & 0,65 \\
\hline Crr. Altavista & 28.973 & 46.642 & 17.669 & 60,98 & 2,27 \\
\hline Crr. San Antonio & 77.007 & 180.773 & 103.766 & 134,75 & 4,06 \\
\hline Total Medellín & 2.343 .049 & 2.724 .051 & 381.002 & 16,26 & 0,72 \\
\hline Comunas & 2.158 .173 & 2.345 .822 & 187.649 & 8,69 & 0,40 \\
\hline Corregimientos & 184.876 & 378.229 & 193.353 & 104,59 & 3,41 \\
\hline Cabecera Medellín & 2.309 .446 & 2.698 .900 & 389.454 & 16,86 & 0,74 \\
\hline Resto Medellín & 33.603 & 25.151 & -8.452 & $-25,15$ & $-1,38$ \\
\hline
\end{tabular}

FUENTE CENSOS DE POBLACIÓN, Y PROYECCIONES MUNICIPALES DEL DANE Y MICRODATOS CENSALES DE IPUMS-INTERNATIONAL, UNIVERSITY OF MINNESOTA. CÁLCULOS PROPIOS

Lo expresado anteriormente es visible en la dinámica de los corregimientos que duplican su población, especialmente San Antonio, Altavista, San Cristóbal e incluso Santa Elena. A pesar de su escaso tamaño poblacional, el corregimiento de Palmitas tendría también un crecimiento importante. Los nuevos asentamientos en 
la cabecera de las zonas rurales de los corregimientos y la ocupación del territorio aledaño a las zonas urbanas de las comunas cercanas permiten proyectar un crecimiento poblacional de los corregimientos de $104,6 \%$ para 2030 , con un ritmo de crecimiento del 3,41\% anual (véase Cuadro 2), proceso que daría lugar a la rururbanización intensiva por vaciamiento desde la saturación urbana del espacio central hacia los contornos exteriores inmediatos.

En este contexto, las comunas tendrían un bajo crecimiento $(8,7 \%$ en las dos décadas), a un ritmo de $0,4 \%$ anual, más bajo que el proyectado para el total de Medellín, pero con aportes importantes en incrementos nominales, que serían cercanos a los registrados en los corregimientos. Si se observa con detenimiento el comportamiento de la dinámica poblacional de las comunas de Medellín en una dirección norte-sur (como aparece en el Cuadro 2), se puede apreciar que hay un contorno espacial demarcado por La Candelaria y Aranjuez, que, junto con Buenos Aires, corresponden a los fragmentos territoriales urbanos con las tendencias más bajas del contorno de las comunas.

Entre las comunas con mayor crecimiento estarían Robledo con 11,38\%, El Poblado con 15,34\%, 12 de Octubre, que junto con Belén sobrepasarían los 200.000 habitantes, Manrique y San Javier con crecimientos cercanos al 8,5\% en el periodo. En los últimos años, El Poblado concentró el 30\% de las construcciones de la ciudad y el $50 \%$ de la inversión en este sector (Sanín, 2010, p. 128), cifras desmedidas, pues su población representa el 5,1\% del total de Medellín.

Resulta claro que existen puntos de presión demográfica desde las comunas hacia los corregimientos. Si consideramos el sur de Medellín, vemos que en el norte las comunas de Robledo, Castilla y 12 de Octubre ejercen presión sobre el corregimiento de San Cristóbal; y en el sur, las comunas Popular, Santa Cruz, Buenos Aires y El Poblado la ejercen sobre Santa Elena, un reto para las instituciones como el Área Metropolitana del Valle de Aburrá (AMva) y la Alcaldía de Medellín, que tienen como deseable el modelo de "ciudad compacta" (Alcaldía de Medellín, 2007; AMva, 2006).

\section{Proyección de la fuerza de trabajo (pet y pea)}

Según los resultados de las proyecciones reflejadas en los mapas de la PET de 2010 en contraste con la de 2030 en el área metropolitana, se obtuvo que, de los 844.883 nuevos habitantes, habría 818.138 en edad de trabajar. De igual forma, se aprecia un aumento en la PET de $1,17 \%$, pero en términos relativos en Medellín, donde su crecimiento es menor al del resto de los municipios, siendo del 0,87 respecto al 1,75\% anual, que es el doble del ritmo de crecimiento de la PET que tendría la capital.

En términos absolutos también el resto de municipios superaría a Medellín, pues dicho aumento llegaría a ser de 424.350 personas respecto a 393.788 personas en edad de trabajar en Medellín, concentrándose dicho crecimiento en las cabeceras de municipios, especialmente de Bello y Envigado. Esta tendencia en la fuerza de trabajo responde a un cambio en la dinámica que el modelo de industrialización le había imputado a Medellín como concentradora industrial en el siglo pasado (Franco, 2006, p. 98), por lo que en la actualidad se aprecia una desconcentración de dicha actividad hacia los territorios vecinos en el área metropolitana. 
En casi todos los municipios se espera que haya un mayor crecimiento de la PET masculina que de la femenina, especialmente en sus cabeceras municipales, aunque en municipios como Itagüí y Sabaneta habría mayor crecimiento relativo de la PET femenina. En Medellín, siguiendo la dinámica poblacional, se espera que haya bajas tasas de crecimiento de la PET en las comunas, principalmente en La Candelaria, Laureles-Estadio, Buenos Aires y Belén, mientras que en sus corregimientos se presentarían elevadas tasas de crecimiento, especialmente en Palmitas, San Antonio y San Cristóbal. De igual forma, en casi todos los corregimientos, con excepción de Altavista y en la comuna de Manrique, se tendría un mayor crecimiento relativo de la PET femenina que de la masculina. A la pregunta “ ¿se quedarán en el campo las generaciones que están creciendo en el corregimiento para reproducir el modo de vida campesino?” (Muñoz, 2010, p. 366), las tendencias demográficas muestran que las nuevas generaciones de la fuerza de trabajo buscan migrar a las cabeceras municipales y a otros centros urbanos a actividades urbanas diferentes a las agropecuarias.

Estas tendencias en la PET de los municipios del área metropolitana configuran el contraste visible en los mapas de la PET de 2010 y 2030 (véase Figura 1). En ellos se destaca la notoria estabilidad de tonalidades en las comunas de Medellín (en escala de grises), respecto al aumento en la intensidad en las cabeceras de los municipios aledaños, como son Bello, Itagüí, Copacabana, Sabaneta y Envigado, y en los corregimientos de Medellín (en escala de grises), confirmando que muchos campesinos migran por oportunidades que ofrece la industria: salario fijo, prestaciones sociales, asistencia médica (Gaviriae et al., 2008, p. 16).

En cuanto a la tendencia de la tasa de dependencia en los municipios del área metropolitana, los niveles seguirán elevados en el núcleo urbano de Medellín y generalizados en casi todas las comunas, con excepción de San Javier y Manrique, cuyas tasas son menores al 50\% (véase Figura 1). En el caso de los corregimientos de Medellín, se presenta una contracción de la tasa de dependencia que lograría reducir de menos de $40 \%$ en 2020 hasta llegar a niveles cercanos al 30\% en 2030. Por sexo, los resultados muestran que habría predominio de mayores tasas de dependencia femenina que masculina en los municipios y en las comunas de Medellín, exceptuando las comunas Popular, Santa Cruz, Buenos Aires y El Poblado, además de los corregimientos de San Cristóbal y Santa Elena.

La única cabecera municipal del resto de municipios que registraría mayor tasa de dependencia masculina que de femenina es Bello, aunque su zona rural también mostraría el mismo fenómeno, como ocurre en las zonas rurales de Barbosa, Caldas; la tasa más alta de todas estaría en Itagüí, con más de 78 puntos porcentuales de diferencia, debido a que para 2030 se obtuvo una tasa de dependencia masculina de $83,3 \%$ respecto a la femenina para el mismo ańo, de $46,9 \%$, derivado del envejecimiento de la población. Ha aumentado, así, la tasa de dependencia en las comunas de Medellín, y disminuido en sus corregimientos y en el resto de municipios. 
FIGURA I | Algunos indicadores demográficos de fuerza de trabajo de Medellín y Valle de Aburra, 2010-2030
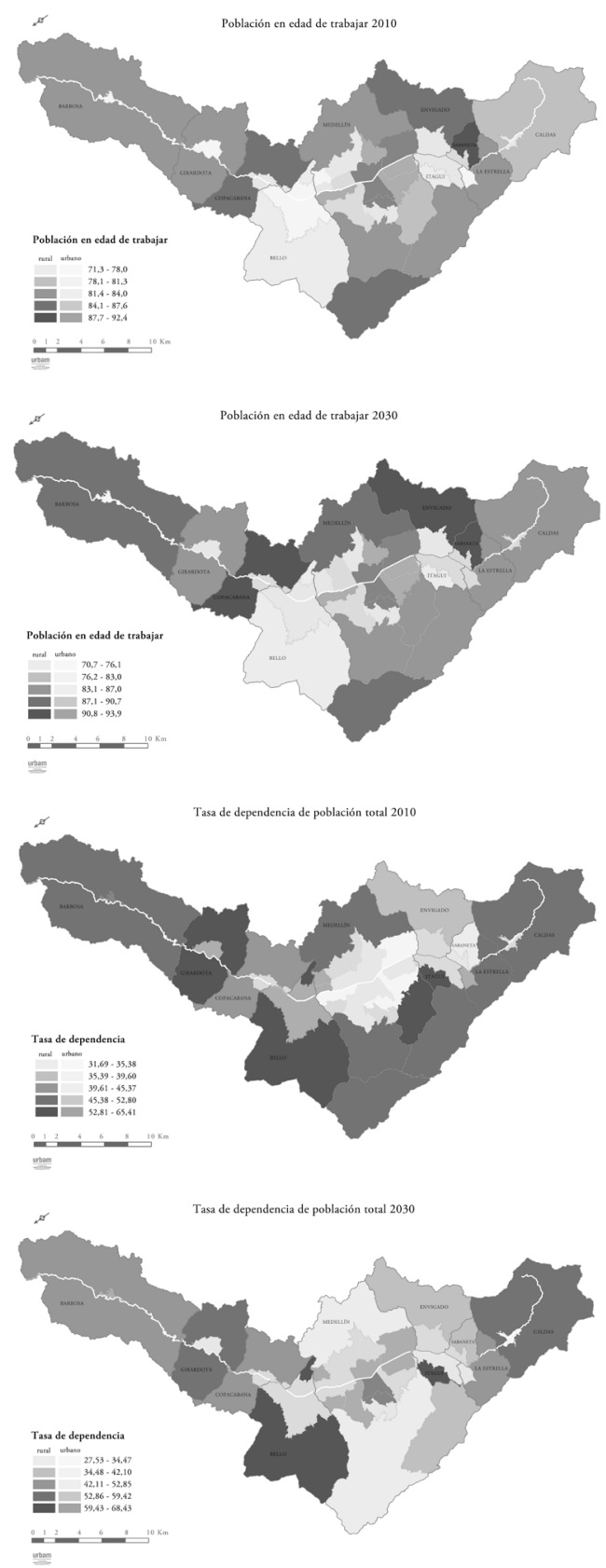

FUENTE CENSOS DE POBLACIÓN, Y PROYECCIONES MUNICIPALES DEL DANE Y MICRODATOS CENSALES DE IPUMS-INTERNATIONAL, UNIVERSITY OF MINNESOTA. CÁLCULOS PROPIOS 
Con los anteriores resultados de las proyecciones de la PET se realizaron las estimaciones de la Población Económicamente Activa (PEA), tomando en cuenta el Cuadro de vida activa para Colombia y la metodología desarrollada por el Centro Latinoamericano y Caribeño de Demografía (CElade). Es de desatacar que, para el caso colombiano, no se realizan proyecciones de PEA para los municipios, por lo que se trató de mantener congruencia entre las tasas de participación históricas como nodo de estimación. Los resultados de la fuerza de trabajo proyectada mostraron una buena estabilidad en los datos, reflejando lo que se apreciaba en las muestras censales DANE-IPUMS de 1964 a 2005 (Minnesota Population Center [MPC], 2011), en la que la tasa neta de participación femenina por grupos quinquenales es menor que la masculina en cerca de 10 puntos porcentuales (véase Gráfico 3).

GRÁfICo 3| Distribución de la población económicamente activa en el Área Metropolitana del Valle de Aburrá, según sexo y grupos de edad, 2005-2030
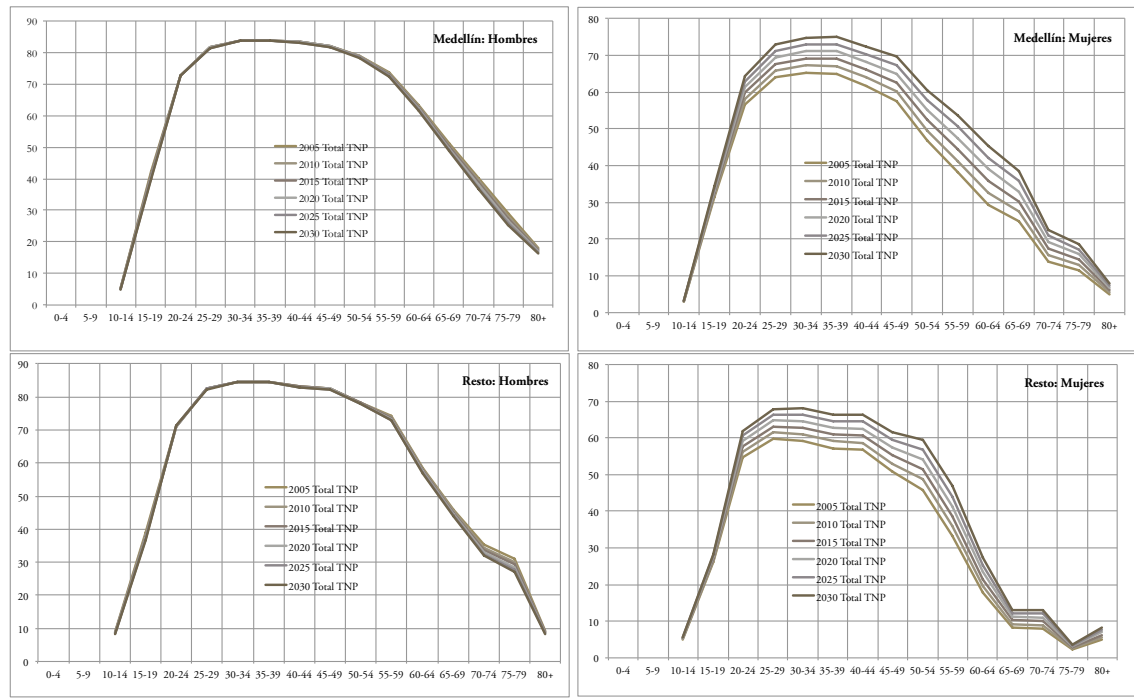

FUENTE CENSOS DE POBLACIÓN, Y PROYECCIONES MUNICIPALES DEL DANE Y MICRODATOS CENSALES DE IPUMS-INTERNATIONAL, UNIVERSITY OF MINNESOTA. CÁLCULOS PROPIOS

La tasa neta de actividad representa la relación entre la Población Económicamente Activa (PEA) y la Población en Edad de Trabajar (PET), que para el área metropolitana era de $55,1 \%$ en 2010 y se espera que pase a ser de $51,5 \%$. Su reducción se debe a las bajas tasas de crecimiento de la PEA en las comunas de Medellín, cuyo ritmo al parecer no superará el 0,3\% anual entre 2010 y 2030, con excepción de Manrique, Robledo y El Poblado. Sus corregimientos, entretanto, tendrían crecimientos superiores al 2\% y hasta al $4 \%$ anual. De allí que los municipios restantes muestren aumentos de su PEA superiores al 1\% y hasta superiores al 2\% anual, como en el caso de Girardota. Las dinámicas se vuelven dramáticas cuando se analizan las tendencias de las tasas netas de actividad de los municipios del área metropolitana. 
Efectivamente, la tasa neta de participación para el área metropolitana caería $6,5 \%$ durante las siguientes dos décadas, debido al proceso de envejecimiento de la PEA que pasa a la inactividad en edades avanzadas a un mayor ritmo que el de la PET. Se presenta así una ineludible disyuntiva de mayor población en edad de trabajar, pero una menor proporción en la actividad (ocupada o desempleada y buscando trabajo). No obstante, el fenómeno de la tendencia negativa de la tasa neta de participación no es nuevo, pues se viene dando desde hace años. Lo novedoso es que su ritmo negativo se desaceleraría en tres puntos porcentuales para la década de 2020 a 2030 , pues pasaría de $-4,8 \%$ a $-1,8 \%$.

Los fragmentos territoriales que arrojarían mayores tasas negativas del crecimiento de la tasa neta de participación serían las comunas de Medellín como Villahermosa, Buenos Aires, La Candelaria, Laureles-Estadio, La América, San Javier, El Poblado, Guayabal y Belén, con caídas de más del 7\% en el periodo, así como el corregimiento de San Antonio, la zona rural de Itagüí y La Estrella, la cabecera municipal de Envigado y Barbosa, y tanto la zona urbana como la rural de Bello.

\section{La tendencia de los jóvenes que no estudian ni trabajan}

El anterior fenómeno en la fuerza de trabajo es uno de los muchos cambios que está teniendo la población activa en el presente siglo. Para profundizar en tales cambios, es relevante colocar la lupa en las estadísticas censales y apreciar uno de los problemas más severos que la sociedad enfrenta. Dentro de la población joven existe un grupo que preocupa en la región por su condición y crecimiento: es el caso de los jóvenes que ni estudian ni trabajan, identificado como los "Ninis", neologismo que es equivalente al acrónimo inglés "NEET", de la expresión "Not in Employment, Education or Training” (Ni trabaja, ni estudia, ni recibe formación).

Inicialmente el concepto de "Nini" surgió en España, acuñado por un grupo de sociólogos que usó dicha etiqueta para destacar a quienes recién salen del colegio y los que ya deberían estar activos en el mercado laboral (Riva Palacio, 2010). Los estudios iniciales del fenómeno de los jóvenes que "ni estudian ni trabajan" en América Latina se concentran en el caso de los jóvenes varones como uno de los indicadores de la creciente marginalización en la sociedad (Kaztman, 1995; Filgueira, 2000); posteriormente dicha tendencia se generalizaría a las mujeres jóvenes, agravando el problema. Por ello, las cifras no son homogéneas en la región latinoamericana, pues en países como Bolivia el grupo en referencia alcanza a ser el 11,4\% de las y los jóvenes, en Ecuador es del 14,7\%, en países como Honduras alcanza al 26,7\% y en Colombia representa el $27,6 \%$ de la población joven, mientras que en Argentina, Brasil, México y Perú las cifras oscilan entre 17,9\% y 20,5\% (D’Alessandre, 2010). En Colombia, las miradas al fenómeno dan cuentan del dramatismo del mismo, pues recientemente se conoció que el $27 \%$ de los jóvenes de escasos recursos de los estratos 1 y 2, ni estudia, ni trabaja, ni busca empleo -cifras referidas por Bruce Mac Master, director del Departamento de la Prosperidad Social (Dps) (Portafolio, 2012)-. De allí la importancia de exponer el fenómeno para una de las áreas metropolitanas más complejas del país, como lo es el Área Metropolitana del Valle de Aburrá. 
$\mathrm{Al}$ rastrear el fenómeno de los "Ninis" a través de las muestras censales de IPUMS desde 1964 a 2005, vemos que se hace visible a partir de 1973; para 1985, el número de jóvenes en esa condición se había reducido en 59,7\% en el periodo censal. Sin embargo, en 1993, con el censo volverían a ser registrados y aumentarían en 37\% para toda el área metropolitana, siendo un poco mayor en la zona urbana y en Medellín y mucho más entre los hombres que entre las mujeres.

\section{GRÁfICO 4| El fenómeno de los “Ninis” (ni estudian ni trabajan) en Medellín según grupos de edad y zonas, 1993-2005}

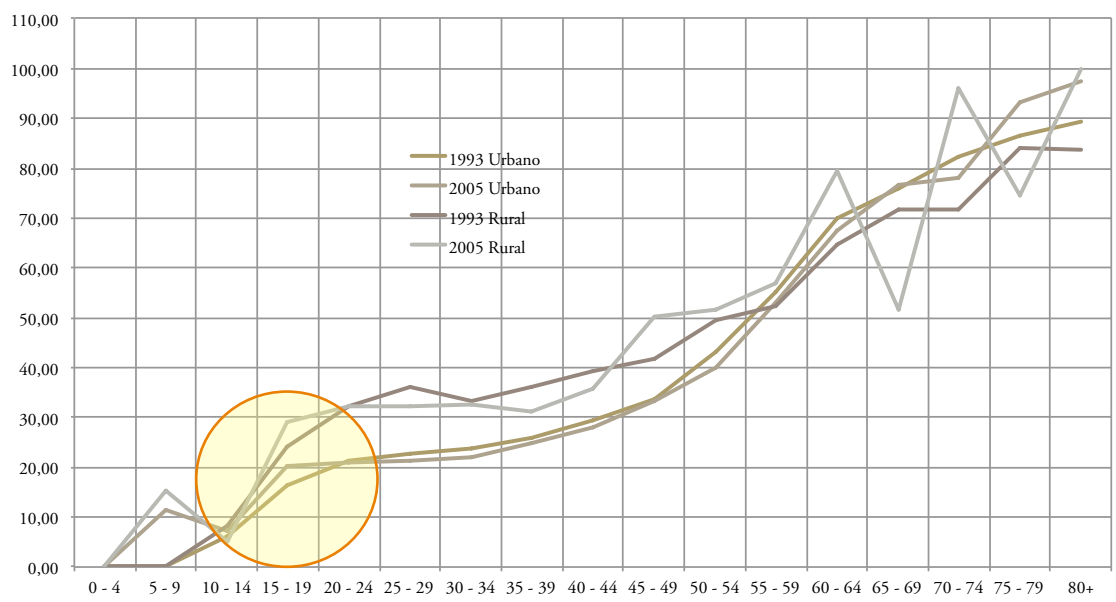

FUENTE CENSOS DE POBLACIÓN, Y PROYECCIONES MUNICIPALES DEL DANE Y MICRODATOS CENSALES DE IPUMS-INTERNATIONAL, UNIVERSITY OF MINNESOTA. CÁLCULOS PROPIOS

Ya en el nuevo censo de 2005 se sigue observando el fenómeno, pero con una notable reducción de 60,5\% respecto al registrado en 1993; lo más destacable fue la reducción de $135 \%$ entre los jóvenes varones. Si bien las cifras referidas dan cuenta de su reducción, lo que es importante destacar también es el aumento en términos relativos durante el último período intercensal. En efecto, el peso relativo del grupo de niños de 10 a 14 ańos que no estudian ni trabajan en el área metropolitana pasó de 6,1 a 6,6\% entre 1993 y 2005, mientras que el grupo de jóvenes de 15 a 19 años que registraron esa condición aumentaría de 16,8 a 19,9\%. En Medellín la situación no cedería, pues pasó de 6,2 a 7,3\% y de 16,7 a 20,4\% en los mismos grupos de niños y jóvenes (véase Gráfico 4), mostrando que el fenómeno aqueja a los municipios en sus zonas urbanas y con mayor intensidad en las zonas rurales, y más a la juventud masculina que a la femenina, lo que la hace más vulnerable a ser caldo de cultivo de procesos de violencia y de actividades ilícitas (Fedesarrollo \& Instituto SER, 1991, p. 17).

Ahora bien, si el grupo de "Ninis" de niños y jóvenes es preocupante, el grupo de adultos jóvenes y adultos en edad activa que son "Ninis" también lo es. En el Gráfico 4 se aprecia cómo, a pesar de que las proporciones del fenómeno en los grupos por 
edad de 20 años a 40 años muestran que se reduce entre 1993 y 2005, los niveles son verdaderamente alarmantes, pues van de $20 \%$ a $30 \%$ en una población a la cual se esperaría encontrar realizando algunos estudios de calificación, o bien en actividades productivas. De allí que el fenómeno constituya ahora una alarma social en la región, en cuanto a las proporciones de los grupos de población involucrados y a la exposición al riesgo de su vulnerabilidad a otros fenómenos sociales de violencia.

\section{Los contornos de crecimiento de la fuerza de trabajo en el Área Metropolitana}

Del análisis de las proyecciones de la fuerza de trabajo en su relación con el territorio se puede apreciar especialmente su comportamiento en la delimitación de los contornos de crecimiento poblacional que se resaltó atrás. En 1925, en su teoría de las áreas concéntricas, Burguess expuso que el crecimiento de la ciudad se da por procesos de concentración, centralización, descentralización, segregación, invasión y sucesión, articulándose en una dinámica de asentamientos productivos de actividad secundaria y terciaria combinada con zonas residenciales, donde el aspecto laboral y el ingreso hacen que se formen núcleos centrales y franjas periféricas (señaladas por Negrete y Salazar, 1986; Sobrino, 1998; y Camagni, 2005), además de formarse un espacio denominado hinterland, que es la zona próxima a ser anexada a la mancha urbana donde se encuentra la franja periurbana (Graizbord \& Salazar, 1986). El papel que desempeńan las comunas en su dinámica viene marcado por la traza que dentro de ellas dibujan los micropoderes, que en su momento histórico de mayor conflicto y violencia intervenían en la cotidianidad de la población (Useche, 1999). Aunque tales actores violentos convirtieron a las comunas en botín de guerra (Álvarez, 2003, p. 330), la actual disminución de las muertes violentas ha permitido que las expectativas de sus pobladores cambien.

Las proyecciones de la PEA acentúan las dinámicas poblacionales encontradas para la población total en el territorio. Los contornos se hacen más evidentes y el crecimiento de la PEA en los corregimientos y municipios del tercer y cuarto contorno son mayores respecto a los que se aprecian en el primer y segundo contorno del área, estos últimos más difusos (Gaviria et al., 2008, pp. 16-20), pues los centros urbanos predominantes inducen mayor fuerza gravitacional en los anillos subsiguientes (Alzate, 2009, p. 17).

En efecto, en el Gráfico 5 se observa el primer contorno interior, donde se encuentran las tasas de crecimiento de la fuerza de trabajo más bajas entre las comunas, como son el 0,01\% y 0,04\% anual de Laureles-Estadio y La Candelaria. El segundo contorno muestra tasas de crecimiento que se alternan entre altas y bajas en las comunas del sur, como son Villa Hermosa, Guayabal y El Poblado respecto a Belén y Buenos Aires, mientras que en las comunas del norte se esperaría un crecimiento de su PEA más homogéneo, con tasas que van del 0,26\% al 0,41\%.

Hacia los corregimientos, los contornos iniciales se acentúan mucho más, como lo muestra el tercer contorno, pero se adicionan a su vez los municipios aledańos, formando un espacio de concentración poblacional y de alto crecimiento de la fuerza de trabajo que se espera siga siendo muy dinámico durante las siguientes dos décadas, con tasas superiores al 2\%, y en algunos casos mayores al 4\%. La presión 
por usos del suelo ha llevado a disminuciones de densidad industrial en Itagüí, Medellín y Sabaneta respecto a incrementos en otros (Ortega, 2010, pp. 58-59).

GRÁfICO 5 Contornos de crecimiento de la población económicamente activa, Área Metropolitana de Medellín, Valle de Aburrá 2010-2030

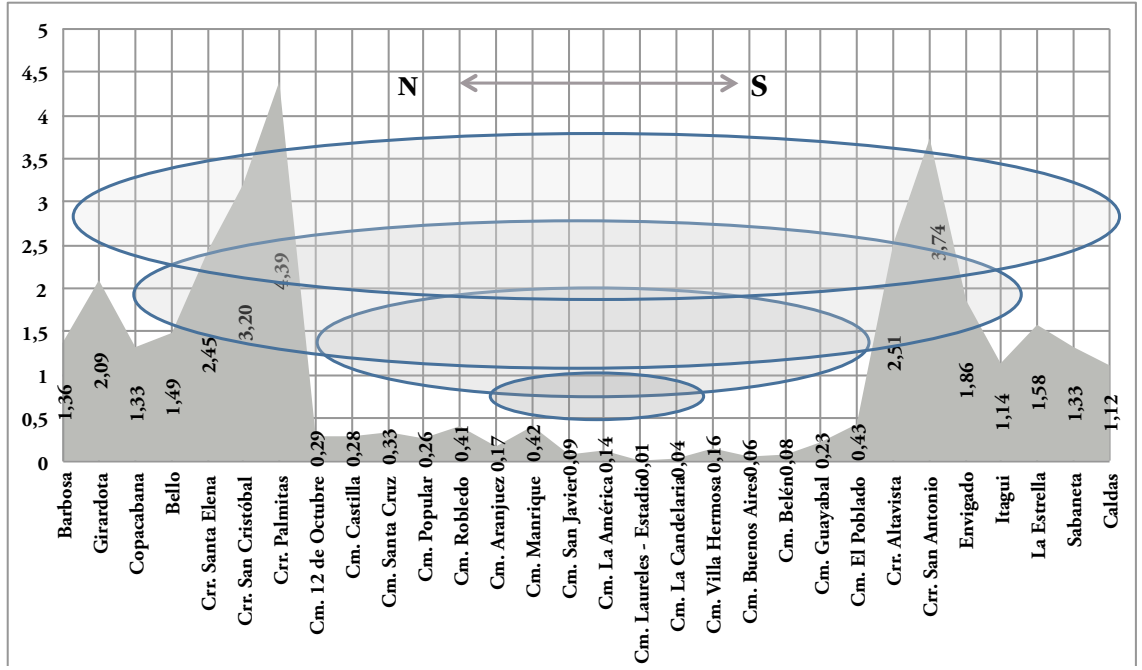

FUENTE CENSOS DE POBLACIÓN, Y PROYECCIONES MUNICIPALES DEL DANE Y MICRODATOS CENSALES DE IPUMS-INTERNATIONAL, UNIVERSITY OF MINNESOTA. CÁLCULOS PROPIOS

El contorno exterior o cuarto contorno estaría mostrando la dinámica de los municipios metropolitanos más alejados del núcleo central, pero exponen un componente nuevo, que sería el fuerte proceso de conformación de dos puntos intermedios, como son las cabeceras municipales de Girardota al sur del área y La Estrella al norte, con tasas de crecimiento de su fuerza de trabajo del orden del 2,1\% y 1,6\% anual, respectivamente. La expansión del área metropolitana seguirá afectando regiones circunvecinas (Gaviria et al., 2008, p. 17) y su dinámica empresarial en Copacabana, Girardota, Sabaneta y Caldas seguirá alta, conviviendo empresas logísticas y de transporte junto a empresas de producción en serie y de organización compleja (Ortega, 2010, p. 63). Los grandes proyectos y la relocalización de actividades productivas en lugares cercanos al área metropolitana han activado la valoración de regiones aledañas, como el Valle de San Nicolás, reconocido por su ubicación geoestratégica en el "Triángulo de oro" y partícipe del "Gran trapecio andino" (Gaviria et al., 2008, pp. 59-60), que se convertiría en un polo de desconcentración y desarrollo.

\section{Los escenarios de crecimiento de la fuerza de trabajo}

Después de realizar las proyecciones de la fuerza de trabajo para el área metropolitana, se propone un ejercicio de estimación de los escenarios en que se podría mover su mercado de trabajo a 2030. Para ello se estableció la estrategia de conformar dos 
escenarios: el primero que reuniera las tendencias de los indicadores del mercado laboral de la última década, y el segundo que fuera un escenario teórico que sirva en la planeación como referente del ideal que se desea alcanzar.

Para estimar el primer escenario del mercado laboral del área metropolitana, se consideró el comportamiento tanto de la tasa de desempleo (TD), como de la tasa de ocupación (TO) y de la tasa neta de participación (TNP) de manera trimestral, desde julio-septiembre de 2001 a octubre-diciembre de 2010. En la serie histórica trimestral de los indicadores se logra observar el efecto que tuvo la crisis mundial iniciada en 2007 y que desembocó en una situación insostenible en 2008, por el aumento de los precios de las materias primas y el efecto dominó que generó la burbuja especulativa del mercado inmobiliario en Estados Unidos, lo que determinó la intervención de los bancos centrales para inyectarle liquidez al sistema bancario tanto de ese país como de las naciones europeas contaminadas. La crisis se extendería por América Latina, con efectos cuya intensidad estuvo relacionada con su grado de dependencia económica, principalmente respecto de Estados Unidos.

Aunque Colombia cuenta con una proporción importante de comercio y negocios con el país del norte, no tiene con él una dependencia económica semejante a la de otros países de la región. Sin embargo, no se puede negar el efecto negativo que tuvo la crisis iniciada en 2007 en el mercado regional y local del área metropolitana, reflejado en el aumento de la tasa de desempleo, que saltó de estar en 11,2\% entre octubre y diciembre de 2007 a un dramático 19\% en el primer trimestre de 2009 (véase Gráfico 6). Incluso, la región Medellín-Valle de Aburrá registró la mayor TD entre las cinco principales ciudades colombianas y sus áreas metropolitanas en el periodo 2009-2011 (Álvarez \& Agudelo, 2011, p. 70).

GRÁfICo 6| Indicadores del mercado laboral del Área Metropolitana del Valle de Aburrá, 2001-2010, para los escenarios a 2030

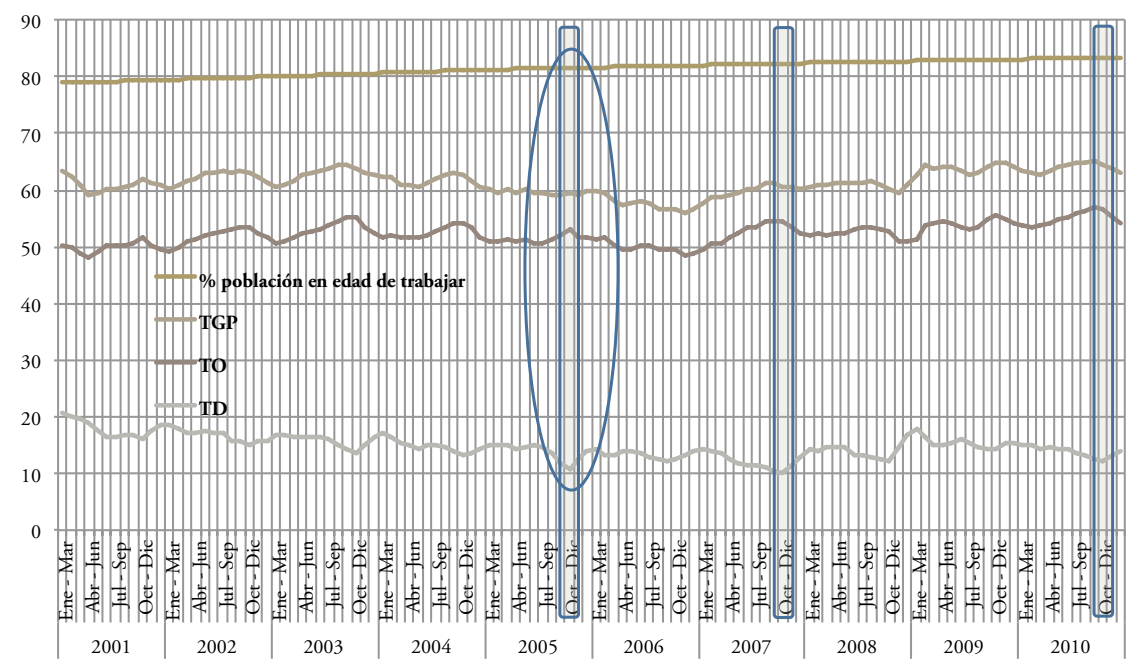

FUENTE DANE - ENCUESTA CONTINUA DE HOGARES, GRAN ENCUESTA INTEGRADA DE HOGARES. SERIE TRIMESTRE MÓVIL, 2OOI-2OIO 
Pese a ello, los efectos de la crisis en la to no resultaron tan bajos como los registrados en el área metropolitana en el cuarto trimestre de 2006. Las inversiones realizadas vía obras en infraestructura, que apuntalaron la política social de los programas de gobierno de ese entonces, lograron generar los empleos que compensaron la caída que estaba teniendo el mercado. Pese al bache que se registrara en el cuarto trimestre de 2008 y que llevaría a la tasa de ocupación a 51\%, el nivel más bajo en el periodo de crisis mundial, la economía del área metropolitana se recuperaría y mantendría en alza la tasa de ocupación.

Con la ayuda del Gráfico 6 es posible identificar los momentos históricos que pueden ser vistos como los mejores, teniendo como parámetro el mercado de trabajo del área metropolitana. De los trimestres de la década para las tres series históricas se seleccionaron tres momentos con baja TD, alta TO y moderada TNP (resaltados con barras verticales). De ellos se descartaron los mejores trimestres de 2007 y 2010, por encontrarse antes y en periodo de crisis y estar afectado por niveles coyunturales con baja estabilidad. Por tal motivo, se seleccionó el escenario de 2005, que se enmarca en un momento censal (resaltado además con elipse), mostrando una TNP de $58 \%$ de la PET que se encuentra activo, con una TD de 11,2\% y una ocupación del $51,6 \%$ de la PET, que son los indicadores que sobresalen como los puntos de mejor comportamiento del mercado de trabajo (mismas cifras citadas por Álvarez y Agudelo, 2011, pp. 73-74).

Con ese momento se extrapoló el escenario como de superación de la crisis económica mundial y sus efectos en la economía regional y local; se procedió a establecer dichos indicadores como los referentes que debieran alcanzarse para el ańo 2030, tomando como punto de partida los indicadores del año 2010. El resultado de la estimación del crecimiento de la PET es de 818.138 entre 2010 y 2030, mientras que el de la PEA es de 346.172 (véase Cuadro 3). Tomando entonces el primer escenario correspondiente a una TD de $11,2 \%$, una To $51,6 \%$ y una TNP de $58 \%$ como ideal para 2030, se tendría que crear 316.077 nuevos empleos, de los cuales 158.368 debieran generarse al 2020 para sostener la tendencia del 52,5\% de la tasa de ocupación y de $11,5 \%$ de la tasa de desempleo. Ello representaría un aumento del desempleo en 18.489 personas a 2020 y 11.605 personas a 2030, con un crecimiento en dos décadas del orden de 30.094 personas. Con esas estimaciones se puede ver que, de los 844.883 habitantes adicionales, 818.138 serían en edad de trabajar; de ellos, 346.172 corresponderían a población activa, siendo el $34,7 \%$ ocupados de esa nueva población en el área metropolitana, que sostendrían al 65,3\% restante de la población adicional.

Para el segundo escenario se tomó como base lo que se conoce en macroeconomía como el nivel de pleno empleo (NAIRU, por sus siglas en inglés: Non-Accelerating Inflation Rate of Unemployment), que se considera como una TD del 5\%. Se reconoce así el nivel de desempleo que no genera inflación en la economía y, por ende, sería el nivel óptimo de alcanzar como siguiente supuesto para establecerlo de base al segundo escenario. Con la tasa de desempleo de 11,6\% registrada en 2010 en el área, para alcanzar el nivel de 5\% para 2030, se estimaría que para 2020 debiera ser de $8,3 \%$, lo que requeriría generar 419.905 nuevos empleos, de los cuales 204.230 tendrían que surgir en la primera década. 
CUADRO 3 | Estimación del crecimiento de la población ocupada y desempleada en el Área Metropolitana bajo dos escenarios, 2010-2030

\begin{tabular}{|c|c|c|c|c|}
\hline CONCEPTO & 2010 & 2020 & 2030 & $2010-2030$ \\
\hline Población Total & 3.544 .703 & 3.994 .609 & 4.389 .586 & \\
\hline Crec. Población Total & & 449.906 & 394.977 & 844.883 \\
\hline Población en Edad de Trabajar & 2.927 .094 & 3.375 .815 & 3.745 .232 & \\
\hline Crec. Población en Edad de Trabajar & & 448.721 & 369.417 & 818.138 \\
\hline Población Económicamente Activa & 1.827 .124 & 2.003 .981 & 2.173 .296 & \\
\hline $\begin{array}{l}\text { Crec. Población Económicamente } \\
\text { Activa }\end{array}$ & & 176.857 & 169.315 & 346.172 \\
\hline \multicolumn{5}{|c|}{ Escenario de Óptimos Indicadores de la Década } \\
\hline TNP & 62,4 & 59,4 & 58,0 & \\
\hline Tо & 55,2 & 52,5 & 51,6 & \\
\hline TD & 11,6 & 11,5 & 11,2 & \\
\hline Ocupados & 1.614 .632 & 1.773 .000 & 1.930 .709 & \\
\hline Crec. Ocupados & & 158.368 & 157.710 & 316.077 \\
\hline Desempleados & 212.492 & 230.982 & 242.587 & \\
\hline Crec. Desempleados & & 18.489 & 11.605 & 30.094 \\
\hline \multicolumn{5}{|c|}{ Escenario de Pleno Empleo (NAIRU) } \\
\hline TD Natural o de Pleno Empleo $\left({ }^{*}\right)$ & 11,6 & 8,3 & 5,0 & \\
\hline Desempleados con Pleno Empleo $\left(^{*}\right)$ & 212.492 & 166.630 & 108.665 & \\
\hline $\begin{array}{l}\text { Crec. Desempleados con Pleno } \\
\text { Empleo }\left(^{*}\right)\end{array}$ & & -45.863 & -57.965 & -103.828 \\
\hline Crec. Ocupados con Pleno Empleo $\left(^{*}\right)$ & & 204.230 & 215.675 & 419.905 \\
\hline
\end{tabular}

(*) NAIRU (NON-ACCELERATING INFLATION RATE OF UNEMPLOYMENT, SIGLAS EN INGLÉS CORRESPON-

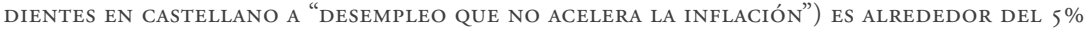

FUENTE CENSOS DE POBlación, DANE Y MiCRodatos CENSAles de IPUMS-INTERNATIONAL, UNIVERSITY OF MINNESOTA. DANE - ENCUESTA CONTINUA DE HOGARES, GRAN ENCUESTA INTEGRADA DE HOGARES. CÁLCULOS PROPIOS

Alcanzar el nivel de pleno empleo en la economía local le significaría a su estructura productiva un esfuerzo que le garantizara trabajo digno al $49,7 \%$, y le permitiera generar los recursos necesarios para sostener con una satisfactoria calidad de vida al restante $50,3 \%$ de la población que tendrá el área a 2030. Implicaría aumentar en $32,8 \%$ el esfuerzo en recursos necesarios para pasar del primero al segundo escenario. Ello podría ser posible si se mantuviera la desconcentración de la actividad productiva en la región central del Valle de Aburrá (Ortega, 2010, pp. 43-44) registrada en la primera década del siglo xxI, mostrando la transición productiva de un territorio de crecimiento medio y bajo hacia crecimiento medio y alto (Ortega, 2010, p. 56).

El proceso actual se basa en la ciudad posfordista, que aumenta la participación de la población económicamente activa en trabajos no asalariados -cuenta propia, subcontratados y no remunerados- (Franco, 2006, p. 101), quedando la fuerza de trabajo disponible para el mercado y sin anclaje para localizarse en cualquier lugar del espacio metropolitano, en un encadenamiento productivo socioterritorial 
vertical excluyente (Betancur, Urán \& Stienen, 2001, p. 256), que obliga a identificar políticas activas de empleo locales sin problemas de articulación entre entidades territoriales (Álvarez \& Agudelo, 2011, pp. 79-82).

\section{Conclusiones}

Las proyecciones oficiales del DANE, que llegaban hasta 2015 para las comunas y corregimientos de Medellín y hasta 2020 para los demás municipios, se lograron incorporar a los resultados de las proyecciones de población para el área metropolitana. De acuerdo con dichos resultados, se tiene una estimación de 4.389.585 personas para el área metropolitana, 844.883 personas más entre 2010 y 2030, con un crecimiento de $1 \%$ anual y con mayor dinamismo en la zona urbana de los extremos de la región central del área metropolitana (Bello, Envigado e Itagüí) que en Medellín.

Se estima que la población de Medellín a 2030 será de 2.724 .051 personas, lo que significa un aumento en 381.002 personas entre 2010 y 2030, con ritmos de crecimiento de $0,72 \%$ anual, lo que mostraría un mayor crecimiento en los corregimientos frente al de las comunas. Tal proceso sería ocasionado por la búsqueda que realizaría la nueva población -desde ahora se aprecia el efecto- hacia la zona rural de Medellín y los municipios contiguos.

La estructura poblacional contiene el proceso de envejecimiento que robustece al grupo de edad de entre 30 y 44 años, cuyo efecto es la reducción en la edad activa. Los jóvenes metropolitanos de la primera década serán reemplazados por la población infantil, que demandará más y nuevos servicios de educación y de salud. La presión hacia el mercado de trabajo será mucho mayor por la búsqueda de oportunidades laborales, debido a que los jóvenes de ahora pasarán a ser jóvenes adultos. La tasa neta de participación femenina por grupos quinquenales es menor en cerca de 10 puntos porcentuales a la masculina.

Los "Ninis" constituyen un fenómeno que viene en aumento, especialmente entre los hombres jóvenes, y de mayor intensidad en las zonas urbanas. Sin embargo, resulta de alta preocupación que no se vea un aminoramiento en la población de jóvenes y que ello más bien se extienda entre los adultos jóvenes, pues es una franja vulnerable de la población que alimenta el circuito de actividades delincuenciales. De no ser articulado dicho grupo a procesos de recuperación social, continuará minando los esfuerzos por lograr superar la violencia, que ha podido reducirse pero que sigue como un permanente riesgo social.

Las proyecciones de población en edad de trabajar (PET) mostraron tasas de crecimiento más elevadas que el de la población total, y algo similar ocurrió con la población económicamente activa (PEA). La estimación del crecimiento de la PET sería de 818.138 personas entre 2010 y 2030, mientras que el de la PEA estaría en 346.172 personas. Para el primer escenario, basado en el mejor momento que tuvo el mercado de trabajo del área en la década, se requerirán 316.077 nuevos empleos; y en el segundo escenario, identificado como el óptimo de pleno empleo, se demandarían 419.905 nuevos empleos. 
Finalmente, las proyecciones de población total, PET y PEA, muestran que el área metropolitana tiene contornos de crecimiento y que ellos son clave para la planeación, diseño y formulación de política pública con programas que sean efectivos para acompañar su transformación. En ese sentido, el primer contorno de las comunas centrales de Medellín va acompañado del segundo contorno, que se acerca a las demás comunas en la zona urbana de Medellín, ambos acompańados por el tercer contorno de corregimientos en la zona rural y de los municipios colindantes a la dinámica del centro e inmersos en ella, y que recibe la presión del crecimiento urbano. Adyacente a ellos se encuentra el cuarto contorno, que apenas inicia su tendencia acompańando los cambios de los otros tres contornos. En conjunto, vienen a mostrar los efectos de los fenómenos de rurubanización y de ampliación de la mancha urbana, cuya diferenciación de intensidad modelará en las siguientes dos décadas el Área Metropolitana del Valle de Aburrá.

\section{Referencias bibliográficas}

Alcaldía de Medellín. (2007). Politica pública de desarrollo rural para la ciudad de Medellín. Medellín: Autor.

Álvarez, T. (2003). Medellín: de una pequeña villa a una ciudad violenta. Iatreia. Revista Médica, 16(4), 325-335. En http://www.iatreia.udea.edu.co/index.php/iatreia/article/ view/4013/3728

Álvarez, R. \& Agudelo S. (2011). Políticas locales de empleo en cuatro municipios del Valle de Aburrá, Colombia. Equidad y Desarrollo, 16(2), 53-85. En http://revistas.lasalle.edu. co/index.php/ed/article/view/137/112

Alzate, M. (2009). Procesos de suburbanización en el área de influencia metropolitana de la ciudad de Medellin. Tesis de Maestría, Instituto de Estudios Urbanos. Universidad Nacional de Colombia, Medellín.

Área Metropolitana del Valle de Aburrá. (2006). Directrices Metropolitanas de Ordenamiento Territorial. Hacia una región de ciudades. Acuerdo Metropolitano No 15, noviembre. Medellín: AMva.

Arroyave, B. \& Muñoz, L. (1994). Cambio social y cultural en San Sebastián de Palmitas. Tesis de grado en Antropología. Universidad de Antioquia, Medellín.

Betancur, M., Urán, O. \& Stienen, A. (2001). Cadenas productivas y redes de acción colectiva en Medellín y el Valle de Aburrá. Economía, Sociedad y Territorio, 3(10), 221-259. En https://www.google.com/url?q=http://www.redalyc.org/articulo. oa\%3Fid\%3D11101002

Blanes, A., Gil, F. \& Pérez, J. (1996). Población y actividad en España: evolución y perspectivas. Colección Estudios e Informes, Centre d'Estudis Demogràics n 5. España: La Caixa.

Bolaños, M. (2000). Revisión crítica a la teoría de la transición epidemiológica. Papeles de Población, 6(25), 179-206. En http://www.redalyc.org/pdf/112/11202509.pdf

Busquets, J. (1993). Perspectiva desde las ciudades. Ciudad y territorio. Revista de Estudios Territoriales, 1(95-96), 163-174. 
Caldwell, J. (1990). Introduction. En J. C. Caldwell, S. Findley, P. Caldwell, G. Santow, W. Cosford, J. Braid \& D. Broers-Freeman (Eds.), What we know about health transition. The cultural social and behavioural determinants of health. Canberra: Australian National University.

Camagni, R. (2005). La renta del suelo urbano (o de la sinergia), En R. Camagni, Economía Urbana, pp. 163-177. Barcelona: Antoni Bosh.

Departamento Administrativo Nacional de Estadística (DANE). (2007). Proyecciones nacionales $y$ departamentales de población. 2005-2020. Estudios Postcensales n 7. Bogotá: Autor.

Departamento Administrativo Nacional de Estadística (DANE). (2008). Proyecciones municipales de población: Metodología. 2006-2020. Bogotá: Autor.

D’Alessandre, V. (2010). Adolescentes que no estudian ni trabajan en América Latina. Sistema de Información de Tendencias en América Latina (sITEAL), Instituto Internacional de Planeamiento de la Educación (IIPE) - Organización de Estados Iberoamericanos (oeI) - unesco, Cuadernos 04. En http://www.siteal.iipe-oei.org/sites/default/files/ SITEAL_Cuaderno04_20100511.pdf

Departamento Administrativo de Planeación de Medellín (DAP). (2002). Anuario estadístico metropolitano 2002. Medellín: Alcaldía de Medellín.

Fedesarrollo e Instituto SER. (1991). Indicadores Sociales. Coyuntura Social, n 5 (Dic.), 8-19.

Filgueira, C., Filgueira, F. \& Fuentes, A. (2001). Critical choices at critical age: Youth emancipation and school attainment in Latin America. Inter-American Development Bank Research Network Working paper \#R-432. En http://www.iadb.org/research/ pub_hits.cfm?pub_id=R-432\&pub_file_name=pubR-432.pdf

Franco, V. (2006). Centros de poder económico y político en Medellín: dilemas estructurales y selectividad estratégica. Controversia, 186 (junio), 89-110.

Frenk, J., Bobadilla, J. L., Stern, C., Frejka, T. \& Lozano, R. (1991a). Elements for a theory of the health transition. Health Transition Review, 1(1), 21-38. doi: 10.2307/40608615

Frenk, J., Frejka, T., Bobadilla, J. L., Stern, C., Lozano, R., Sepúlveda, J. \& José, M. (1991b). La transición epidemiológica en América Latina. Boletín de la Oficina Sanitaria Panamericana, 111(6), 485-493. En http://hist.library.paho.org/Spanish/BOL/ v111n6p485.pdf

Galyn, D. \& Kates, J. (1997). Refocusing the lens: epidemiological transition theory, mortality differentials, and the AIDs pandemic. Social Science and Medicine, 44(5), 609-621.

Garza, G. (2003). Politicas urbanas en grandes metrópolis: Detroit, Monterrey y Toronto. México, D.F.: El Colegio de México.

Gaviria, Z. (2009). La expansión urbana sobre las periferias rurales del entorno inmediato a la ciudad Metropolitana. En Soluciones de Postgrado eia, 1(3), 63-74. En http:// revistapostgrado.eia.edu.co/Revista\%20Edici\%F3n\%20N\%BA.3/Soluciones $\% 20$ N3\%20art\%205.pdf

Gaviria, Z., Hermelin, M., Forero, L., Restrepo, L., Pérez, M. A., Arbeláez, L. F., Peláez, P. P. (...) \& Tobón Villegas, J. (2008). Realidades y prospectiva del Valle de San Nicolás. Rionegro: Cámara de Comercio del Oriente Antioqueño. En http://bit.ly/1phVxZr

Graizbord, B. \& Salazar, H. (1986). Expansión física de la ciudad de México. En Gustavo Garza (Coord.), Atlas de la ciudad de México (pp. 120-125). México, D.F.: El Colegio de México. 
Heligman, L., Chen, N. \& Babakol, O. (1993). Shift in the structure of population and deaths in less developed countries. En J. N. Gribble \& S. H. Preston (Eds.), The epidemiological transition. Policy and planning implications for developing countries (pp. 9-41). Washington D.c.: National Academy Press.

Janssen, F. \& Kunst, A. (2004). ICD coding and discontinuities in trends in cause-specific mortality in six European countries, 1950-99. Bulletin of the World Health Organization, 82(12), 904-913. En http://www.ncbi.nlm.nih.gov/pmc/articles/PMC2623106/ pdf/15654404.pdf

Kaztman, R. (1995). Marginalidad e Integración Social. Montevideo: Comisión Económica para América Latina y el Caribe (CEPAL).

Leibenstein, H. (1957). Economic backwardness and economic growth. Nueva York: Wiley Co.

Mackenbach, J. (1994). The epidemiologic transition theory. En Journal of Epidemiology and Community Health, 48(4), 329-331. En http://www.ncbi.nlm.nih.gov/pmc/articles/ PMC1059977/pdf/jepicomh00199-0001b.pdf

Méndez, M., López, L. \& Márquez, L. (2006). Incursión ocupacional rural en escenarios no-agrícolas y urbanos: reflexiones en torno a la evidencia empírica. Cuadernos de Desarrollo Rural, 3(56), 117-135. En http://revistas.javeriana.edu.co/index.php/ desarrolloRural/article/view/1238/730

Meslè, F. \& Vallin, J. (2006). The health transition: Trends and prospects. En G. Caselli, J. Vallin \& G. Wunsch (Eds.), Demography: Analysis and synthesis. A treatise in population studies (Vol. 2, pp. 29-44). Nueva York: Academic Press.

Minnesota Population Center (2011). Integrated Public Use Microdata Series, International (Version 6.1) [Colombia database 1993-2005]. En https://international.ipums.org/ international/

Miró, C. (2003). Transición demográfica y envejecimiento demográfico. En Papeles de Población, 9(35), 9-29. En http://redalyc.org/articulo.oa?id=11203502

Moreno, C. (2005). Criterios ambientales para la vivienda y el hábitat en el Valle de Aburrá. Serie Investigaciones CEHAP 23. Medellín: Universidad Nacional de Colombia y AMva.

Muñoz, L. (2010). Nuevos escenarios para la comunidad campesina de San Sebastián de Palmitas luego de la construcción de la Conexión Vial Aburrá-río Cauca y el Túnel de Occidente. Boletin de Antropología, 24(41), 353-375. En http://www.redalyc.org/ articulo.oa?id=55716976016

Naciones Unidas (2005). Handbook on the Collection of Fertility and Mortality Data. Department of Economic and Social Affairs Statistics Division. ST/ESA/STAT/ SER.F/92. En http://unstats.un.org/unsd/demographic/standmeth/handbooks/ Handbook_Fertility_Mortality.pdf

Negrete, M. \& Salazar, H. (1986). Zonas metropolitanas en México, 1980. Estudios Demográficos y Urbanos, 1(1), 97-125. En http://codex.colmex.mx:8991/exlibris/aleph/a18_1/ apache_media/8S53TQ5EN5R31BI5HHQDVI8AEKFH1B.pdf

Notestein, F. (1945). Population: the long view. En E. Schultz (Ed.), Food for the World (pp. 36-57). Chicago: The University of Chicago Press.

Olshansky, J. \& Ault, B. (1986). The fourth stage of the epidemiological transition: the age of delayed degenerative diseases. The Milbank Memorial Fund Quarterly, 64(3), 355-391.

Omran, A. (1971). The epidemiological transition: a theory of the epidemiology of population change. The Milbank Memorial Fund Quarterly, 49(4), 509-538. 
Omran, A. (1998). The epidemiological transition theory revisited thirty years later. World Health Statistics Quarterly, 51, 99-119.

Ortega, G. (2010). Diversificación e inestabilidad productiva un análisis para el Valle de Aburrá (1995 2005). Tesis de Maestría, Instituto de Estudios Urbanos. Universidad Nacional de Colombia, Medellín.

Portafolio (2012, agosto 6). Jóvenes pobres, no estudian, no trabajan, ni buscan empleo. Diario Portafolio. Consultada el 15 de enero de 2013, de http://www.portafolio.co/economia/ jovenes-pobres-no-estudian-no-trabajan-ni-buscan-empleo

Riva Palacio, R. (2010, febrero 17). La generación perdida. Diario El País, Sección Internacional. Consultada el 15 de enero de 2013, de http://www.elpais.com/articulo/internacional/ generacion/perdida/elpepuint/20100217elpepuint_7/Tes

Rogers, R. \& Hackenberg, R. (1987). Extending epidemiological transition theory: A new stage. Biodemography and Social Biology, 34(3-4), pp. 234-243.

Sanín, P. (2010). ¿De ciudad abierta a ciudad cerrada? Configuraciones socio-espaciales en el barrio El Poblado, Medellín. Territorios, 23, 123-142. En http://www.redalyc.org/ pdf/357/35715584006.pdf

Sobrino, J. (1998). Estructura física y etapas de metropolitanismo de la Ciudad de México. México, D.F.: El Colegio de México.

Sobrino, J. (2003). Competitividad de las ciudades de México. México, D.F.: El Colegio de México.

Unikel, L. (1978). El desarrollo urbano de México. México, D.F.: El Colegio de México.

Useche, O. (1999). Coordenadas para trazar un mapa de la violencia urbana en Colombia. Nova y Vetera, 36, 4-17.

Villegas, A. (2005). Discurso sobre la planificación rural en el municipio de Medellín: entre los impactos y los abusos. Boletín de Antropología, 19(36), 149-163. En http://www. redalyc.org/pdf/557/55703607.pdf

Zuluaga, G. (2000). Nueva ruralidad: usos, funciones y actividades. Dinámicas y problemáticas. Enfoques y metodologías sobre el hábitat: memorias de experiencia pedagógica. Ensayos Forhum, 15, 1-12. En http://www.bdigital.unal.edu.co/5015/1/For15-5-Zuluaga. PDF 